\title{
Outbursting Young Stellar Object PGIR 20dci in the Perseus Arm
}

\author{
Lynne A. Hillenbrand ${ }^{1}$, Kishalay De ${ }^{1}$ (1) , Matthew Hankins $^{2}$ (1), Mansi M. Kasliwal ${ }^{1}$ (1), Luisa M. Rebull ${ }^{3}$ (1), Ryan M. Lau ${ }^{4}$, \\ Roc M. Cutri ${ }^{3}$ (D), Michael C. B. Ashley ${ }^{5}$ (D), Viraj R. Karambelkar ${ }^{1}$ (D), Anna M. Moore ${ }^{6}$, T. Travouillon ${ }^{6}$ (D), and A. K. Mainzer ${ }^{7}$

\begin{abstract}
We report the discovery of a likely outbursting Class I young stellar object, associated with the star-forming region NGC 281-W (distance $\sim 2.8 \mathrm{kpc}$ ). The source is currently seen only at infrared wavelengths, appearing in both the Palomar Gattini InfraRed $(1.2 \mu \mathrm{m})$ and the Near-Earth Object Wide-field Infrared Survey Explorer (3.4 and $4.6 \mu \mathrm{m})$ photometric time-domain surveys. Recent near-infrared imaging reveals a new, extended scattered light nebula. Recent near-infrared spectroscopy confirms the similarity of PGIR 20dci to FU Ori-type sources, based on strong molecular absorption in $\mathrm{CO}, \mathrm{H}_{2} \mathrm{O}$, and $\mathrm{OH}$; weak absorption in several atomic lines; and a warm wind/ outflow as indicated by a P Cygni profile in the He I $\lambda 10830$ line. This is a rare case of an FU Ori star with a wellmeasured long-term photometric rise before a sharper outburst, and the second instance of an FU Ori star with a documented two-step brightening in the mid-infrared.
\end{abstract}

Unified Astronomy Thesaurus concepts: Young stellar objects (1834); Circumstellar disks (235); Stellar accretion disks (1579); Pre-main sequence stars (1290)

\section{Introduction}

Episodic accretion in young stellar objects is an important aspect of mass accumulation in stars. It also plays a determining role in the evolution of circumstellar disks and likely influences the process of planet formation, as well as planetary survival. Armitage (2019) provides a review of disk physics in young stars, including nonsteady accretion and disk instabilities.

In "classical" $\mathrm{T}$ Tauri-type young stars, the accretion process is controlled mainly by physics in the inner disk and magnetospheric region that connects the disk to the star. Observed time variability in broadband photometry and in emission-line profiles of these sources is dominated by stochastic behavior, with timescales from hours to days (e.g., Costigan et al. 2014; Findeisen et al. 2015; Cody \& Hillenbrand 2018). The empirical zoo of accretion-related photometric behavior has been measured in unprecedented detail for young stars by space missions such as CoRoT, Spitzer, Kepler, and now TESS, all of which sample timescales from minutes to a few months. Ground-based surveys such as the All-Sky Automated Survey for SuperNovae (ASASSN; Shappee et al. 2014), Palomar Transient Factory (PTF; Law et al. 2009), Zwicky Transient Facility (ZTF; Bellm et al. 2019; Graham et al. 2019), and Asteroid Terrestrial-impact Last Alert System (ATLAS; Tonry et al. 2018) in the optical and VVV (Minniti et al. 2010) in the infrared, by contrast, typically have lower precision and cadence ( 1 day to a few days) but the advantage of long-duration monitoring. Gaia and WISE+NEOWISE also provide precise space-based photometric data, but at much lower cadence (months), with total duration now exceeding a decade. Many of these surveys have all-hemisphere or even all-sky coverage, enabling rare event detectionincluding episodic accretion outbursts in very young stars.
Accretion-driven outbursts associated with young stellar objects have been identified at an increasing rate over the past decade. The discoveries have come from both the ground-based and the spaced-based platforms. Infrared surveys have the advantage of being able to penetrate the high levels of extinction toward the molecular clouds where young stars are born, and to some extent the additional circumstellar extinction of the youngest self-embedded sources.

Although young star outbursts appear to come in a continuum of amplitudes and rise times, for historical reasons (Herbig 1977), the labels EX Lup-type bursts (also known as EXors) and FU Ori-type outbursts (also known as FUors) are often applied. The former are more frequent, characterized by brightness increases of a few magnitudes in amplitude, lasting a few months to $1-2 \mathrm{yr}$, and have emission-line spectra. The latter are more infrequent and can be up to 5-6 mag in amplitude, lasting decades to centuries, and with absorptiondominated spectra except for P Cygni structure in some wind lines. An increasing number of objects are unable to be cast neatly into either of these categories, however. Our knowledge of the spectrum of amplitudes, durations, and duty cycles for young star outbursts is still in its infancy.

The large-scale FU Ori outburst events are intrinsically rare. Furthermore, those few that have been captured in progress have not been particularly well characterized. ${ }^{8}$ The most recent FU Ori outburst discoveries are improving the situation, e.g., Gaia 17bpi (Hillenbrand et al. 2018), Gaia 18dvy (Szegedi-Elek et al. 2020), and NWISE-F J213723.5+665145 (Connelley \& Reipurth 2020; Stecklum 2020). The total census

\footnotetext{
8 The situation can be compared to shorter-timescale rare occurrences, such as tidal disruption events and nearby supernovae, which garner armies of dedicated followers.
} 
of FU Ori-type stars numbers $<30$ objects at present, with less than half of these having had their outburst actually observedrather than inferred from imaging at well-separated preoutburst and post-outburst epochs.

We report here on the discovery of substantial photometric brightening of an object designated as PGIR 20dci. This source is located at R.A. $=00: 52: 20.21$, decl. $=+56: 34: 03.9$, and we associate it with the NGC $281-\mathrm{W}$ star-forming region. The object appears to be a bona fide FU Ori star that has just reached a plateau phase in its brightening.

\section{Source Environment}

NGC 281, also known as Sh 2-184, is an H II region located in the Perseus spiral arm. While the traditional distance in the literature on the stellar population is $\sim 2.2 \mathrm{kpc}$, astrometry from Gaia DR2 (Gaia Collaboration et al. 2018) indicates a somewhat farther $2.8 \mathrm{kpc}$. This is also the distance derived by Sato et al. (2008) from parallax studies at radio wavelengths of a maser population associated with NGC 281-W (where PGIR $20 \mathrm{dci}$ is located). These results place the region on the far side of the Perseus arm, and about $300 \mathrm{pc}$ out of the Galactic plane.

IC $1590(<2 \mathrm{Myr})$ is the main stellar cluster within the nebular region, and contains the bright ionizing sources collectively designated as HD 5005. This source is now a spatially resolved multiple-star system, consisting of components with spectral types 06.5, O8, and O9 (Guetter \& Turner 1997). The cluster thus draws analogies in the literature as a Trapezium-type system. The stellar population of the IC 1590 cluster was investigated by Guetter \& Turner (1997) and Sharma et al. (2012), the latter of whom find a size $r \approx 6.5 \mathrm{pc}$.

To the southwest of the main cluster IC 1590 is a dark lane known as NGC 281-W. Another dark area just to the east of the nebulosity is designated NGC 281-E. These are the locations of molecular clouds at the same distance as the large nebula, and presumably situated, at least in part, on its near side. APOD provides an optical picture ${ }^{9}$ that may clarify the geometry for some readers. CO maps of the overall region can be found in Elmegreen \& Lada (1978) and Lee \& Jung (2003), who provide evidence for cloud compression coming from the direction of the stellar cluster and H II region.

The NGC 281-W molecular core has a bright submillimeter and far-infrared source, readily apparent in Herschel/SPIRE (Griffin et al. 2010) and SCUBA-2 (Holland et al. 2006) maps. This dust clump harbors a collection of mid-infrared point sources to its east and southeast, as seen in WISE (Wright et al. 2010) images.

The portion of this highly embedded cluster that is visible in the near-infrared was discovered and studied by Carpenter et al. (1993). These authors targeted the region with early nearinfrared imaging capabilities based on the position of IRAS $00494+5617$. They found a cluster size $r=0.74 \mathrm{pc}$ (corrected here for differences in the distance assumption), with northeastern and southwestern portions separated by a ridge of high extinction. The "typical" extinction toward the embedded sources was estimated at $A_{V} \approx 10 \mathrm{mag}$, with peak $A_{V} \approx 45 \mathrm{mag}$. Carpenter et al. (1993) also derived a mass of $340 M_{\odot}$ (corrected here for differences in the distance assumption), for the associated CS core. ${ }^{10}$ The same area of NGC $281-\mathrm{W}$ was

\footnotetext{
9 https://apod.nasa.gov/apod/ap110825.html

10 A distance-corrected mass in $\mathrm{CO}$ of $4000 M_{\odot}$ was also reported by Carpenter et al. (1993), though the NGC 281-W cloud is somewhat larger than the area studied, with Lee \& Jung (2003) finding a total mass for the extended cloud of $(1-3) \times 10^{4} M_{\odot}$.
}

specifically targeted in a later similar study by Megeath \& Wilson (1997) using more sensitive radio and infrared observations.

The object of interest here, PGIR 20dci, is located within the region of these previous surveys, about $30^{\prime \prime}$ west of the embedded cluster center. Figure 1 shows a portion of the field imaged by Megeath \& Wilson (1997), with the position of PGIR 20dci designated. Although faintly visible at $K^{\prime}$ band in this archival image, PGIR 20dci has not been previously cataloged, studied, or characterized.

\section{Outburst Discovery and Validation}

In this section, we describe the initial photometric alert that drew our attention to PGIR 20dci. We then detail the optical, near-infrared, and mid-infrared photometric forensics work leading to establishment of the outburst profile over time.

\subsection{Gattini $1.2 \mu \mathrm{m}$ (J-band) Light Curve}

The Palomar Gattini InfraRed survey (PGIR; Moore \& Kasliwal 2019; De et al. 2020a). observes the sky every two nights in the near-infrared $J$ band, to a median depth of $J=15.7 \mathrm{mag}(\mathrm{AB})$. The faint limit is much brighter than this in crowded or confused regions, such as near the Galactic plane and in high surface brightness regions, such as NGC 281-W.

On 2019 August 31, a "hostless" source was first detected in PGIR as a positive image subtraction and flagged (by coauthor K.D.) for photometric and spectroscopic follow-up. The initial source position required some refinement owing to the $8^{\prime \prime}$ pixel size of PGIR. There are no counterparts in optical (e.g., PanSTARRS; Flewelling et al. 2020) or near-infrared (Two Micron All Sky Survey (2MASS); Skrutskie et al. 2006) survey catalogs, though a hard stretch of the 2MASS images does reveal a faint detection at $K_{s}$ and perhaps $H$. A nearby WISE catalog source at 00:52:20.41, $+56: 34: 03.3$ was determined to be the source that had brightened. The brightening is also recorded in NEOWISE (Mainzer et al. 2014) data. The consequent increase in signal-to-noise ratio $(\mathrm{S} / \mathrm{N})$ likely yields better astrometry and hence a more accurate true source position of 00:52:20.21, $+56: 34: 03.9$, which we adopt. This position is 1." 3 away from the position reported for the fainter WISE source, and $4 "-5$ " from the PGIR position.

Figure 2 shows the light curve produced from the PGIR forced photometry pipeline. Point-spread function (PSF) fit photometry is performed, with noise uncertainty at the location of the transient estimated, as described in De et al. (2020b), from difference images produced using the ZOGY algorithm (Zackay et al. 2016).

Since its discovery at $J=14.97 \mathrm{mag}$, PGIR 20dci has slowly increased in brightness. The brightening trend observed during the 2019-2020 season (Figure 2) can be fit with a linear slope of $-0.116 \pm 0.011 \mathrm{mag}$ month $^{-1}$. During the latter part of 2020, the light curve appears to have plateaued, and a linear fit is consistent with zero slope.

\subsection{Prediscovery Photometric Information}

In this section, we discuss the limited photometric record available for PGIR 20dci. 

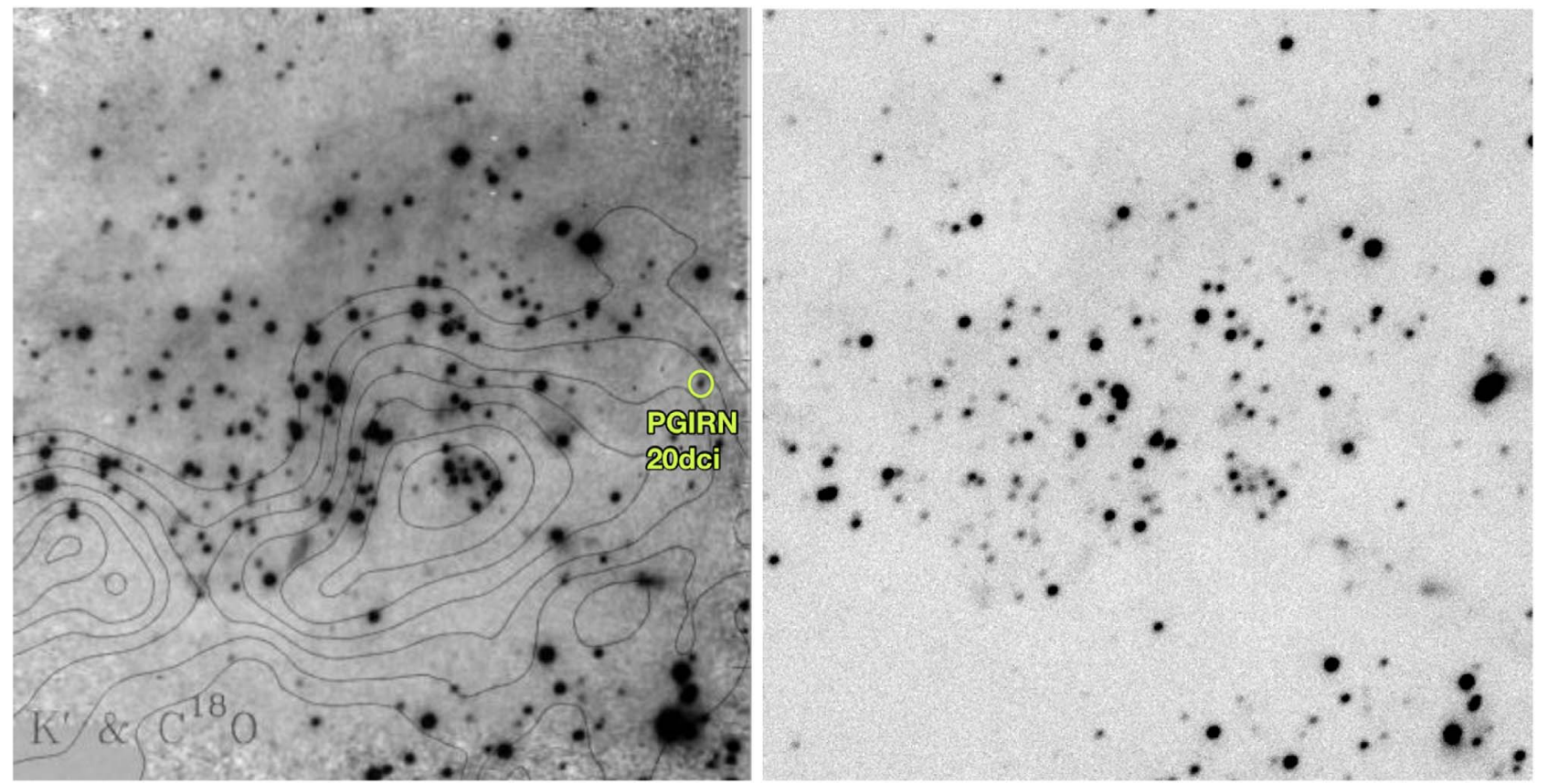

Figure 1. Left panel: figure reproduced from Megeath \& Wilson (1997, their Figure 9) showing a deep near-infrared image taken in $K^{\prime}(2.2 \mu \mathrm{m})$ along with contours of the $\mathrm{C}^{18} \mathrm{O}$ molecular line emission intensity. Faint tick marks along the right axis indicate $10^{\prime \prime}$ intervals, with a total field of view of $2^{\prime} .07 \times 2$ ! 07 . Overlaid in bright green is a marking for the position of PGIR 20dci, which does appear as a faint source in this early 1990s image. Right panel: $K_{s}(2.2 \mu \mathrm{m})$ image obtained in 2020 September, showing a much brighter and extended PGIR 20dci, even though the image is shallower than the one on the left. In both panels, the data are from infrared cameras on the Palomar 200" telescope (PFIR = Prime Focus InfraRed, on the left, and WIRC on the right).

\subsubsection{Optical and Near-infrared}

At the time of its PGIR detection, PGIR 20dci was invisible in optical time-domain survey data, including ASAS-SN and ZTF. Recently, however, a faint optical source with $r=20-20.5 \mathrm{mag}$ is transiently present in ZTF alerts (Masci et al. 2019). There is not a useful optical light curve, however, due to infrequent detection and large error bars.

We also note that PGIR 20dci was undetected in deeper imaging of the NGC 281-W region with Hubble Space Telescope in the optical F814W or F675W filters, from 2000 September, accessed via the Hubble Legacy Archive. PGIR $20 \mathrm{dci}$ is also undetected in archival Keck/DEIMOS images of the region from 2010 October 6.

In the near-infrared, PGIR 20dci was undetected by 2MASS (Cutri et al. 2003), as noted above. While the nominal 2MASS survey limit is $J<15.8$, we derive a more accurate number for this exact environment by considering the error versus magnitude distribution of sources within $4^{\prime}$ of PGIR 20dci. We find that $10 \sigma$ photometry is available to $J=16.1 \mathrm{mag}$ and $5 \sigma$ photometry to $J=17.1 \mathrm{mag}$. While the diffuse background is high in the NGC 281-W region, the dark cloud renders the point-source background and confusion lower than would be the case otherwise. Thus, the 2MASS magnitude limit is plausibly deeper than nominal. We conclude that a reasonable flux upper limit for PGIR 20dci at epoch 2000 January 13 is approximately $J>17.1 \mathrm{mag}$.

In the deeper $J$-band imaging of Megeath \& Wilson (1997), PGIR 20dci is also not apparent, except for perhaps a faint low surface brightness smudge near the edge of detection. The claimed faint point-source limit of these data is $J \approx 21 \mathrm{mag}$. PGIR 20dci does, however, have an apparent faint counterpart in the $K^{\prime}$-band imaging of Megeath \& Wilson (1997), as indicated in Figure 1. Although the sources from this study are not cataloged, the authors report that the fainter stars in the displayed image have $K^{\prime} \approx 18.5$ mag ( $80 \%$ completeness level). PGIR 20dci thus appears to have experienced a $\sim 5$ mag brightening since the mid-1990s epoch of the Megeath \& Wilson (1997) data acquisition.

\subsubsection{Mid-infrared Spitzer}

In the mid-infrared, there is also a faint counterpart to PGIR 20dci in Spitzer/IRAC (Fazio et al. 2004; Werner et al. 2004) imaging. The source appears blue in comparison to the embedded cluster approximately $30^{\prime \prime}$ to the east of PGIR 20dci, perhaps due to somewhat lower extinction away from the center of the molecular core (see contours in Figure 1).

There are three relevant Spitzer/IRAC AORs, one for P.I. Fazio in 2004 (AORKEY 4127744) and two for P.I. Wolk in 2009 (AORKEYs 34780160 and 34780416). The former, from Spitzer's cryogenic phase, includes all four IRAC bands (3.6, $4.5,5.8$, and $8 \mu \mathrm{m})$. The latter two were taken in the post-cryo phase of Spitzer and thus include only IRAC1 $(3.6 \mu \mathrm{m})$ and IRAC2 $(4.5 \mu \mathrm{m})$. We performed aperture photometry at the target's location in the pipeline-produced post-BCD mosaics using a radial aperture of 3 native pixels $\left(1.2\right.$ pixel $\left.^{-1}\right)$ and a background annulus from 3-7 native pixels. Aperture corrections were applied as appropriate to the cryo and the post-cryo observations, as described in the IRAC Instrument Handbook. ${ }^{11}$ The Instrument Handbook also has the zero points ${ }^{12}$ we used for converting the flux densities to magnitudes. Our photometric results for Spitzer/IRAC are provided in the first three rows of Table 1 .

\footnotetext{
11 https://irsa.ipac.caltech.edu/data/SPITZER/docs/irac/iracinstrumenthand book. Specifically, we used values of $1.124,1.127,1.143$, and 1.234 for the four channels in the cryogenic era, respectively, and 1.125 and 1.120 for the two channels in the post-cryo era, respectively.

$12280.9,179.7,115.0$, and 64.13 Jy for the four IRAC channels, respectively.
} 

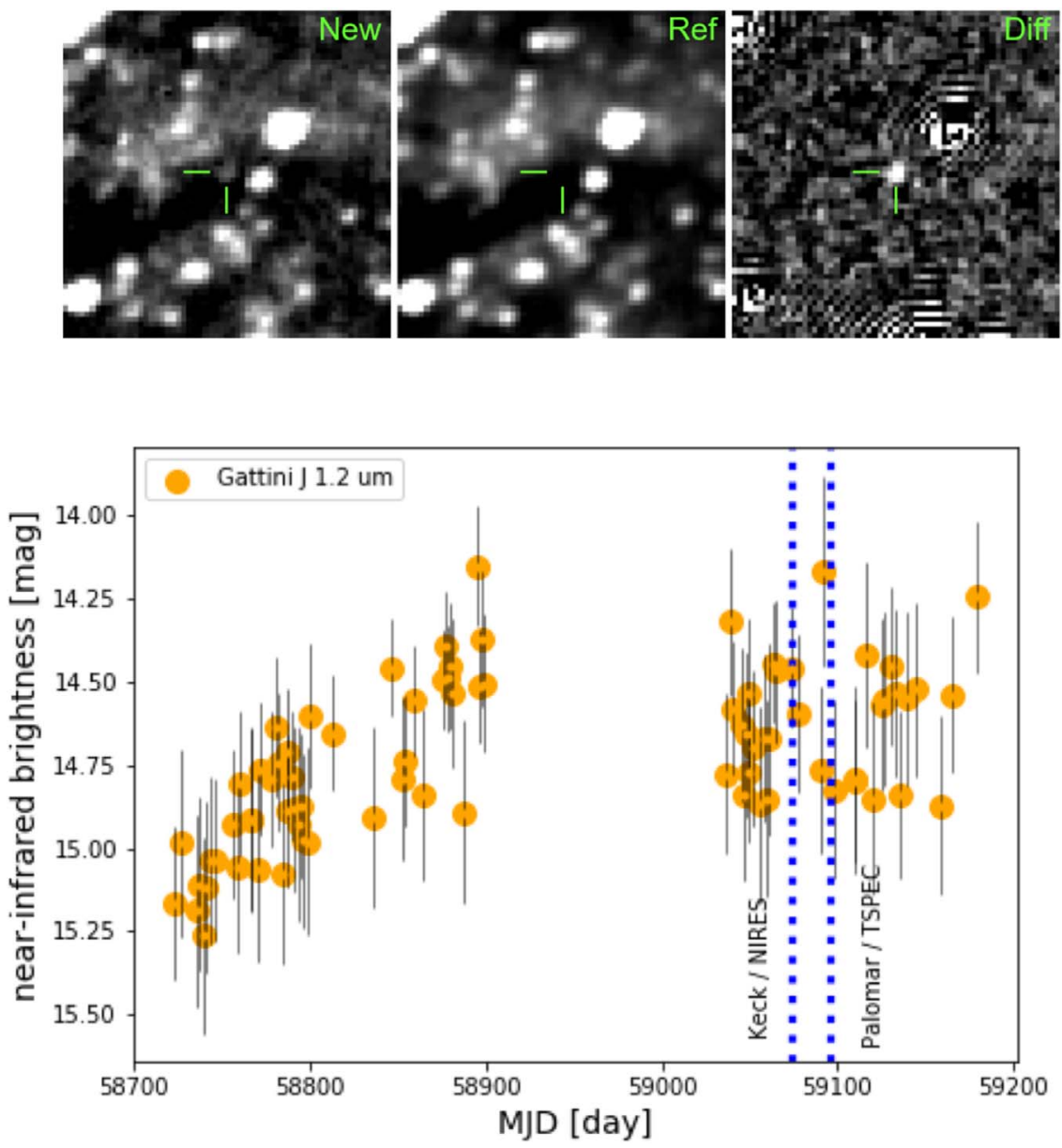

Figure 2. Top panels: discovery imaging sequence showing a $4^{\prime} \times 4^{\prime}$ region centered on PGIR 20dci; orientation is the standard north upward and east to the left. From left to right are the following: the PGIR image from 2019 October 31 (MJD = 58787), the static reference image of the field created from a series of earlier images taken between 2018 October 28 and 2019 January 29, and the subtracted difference image. PGIR 20dci is clearly visible in the last panel and is marked in all three. Bottom panel: $J$-band light curve of PGIR 20dci during 2019 and 2020. The observed upward slope in the data points at MJD $<59000$ corresponds to a source brightening rate of $-0.116 \pm 0.011 \mathrm{mag} \mathrm{month}^{-1}$. The light curve then flattens, and a fit for MJD $>59000$ is consistent with zero slope ( $-0.017 \pm 0.022 \mathrm{mag}$ month $^{-1}$ ). We note that $J$-band observations from much earlier indicate a significantly fainter source, with estimated limits $J>17.1$ mag (2MASS) and $J>20$ mag (Megeath \& Wilson 1997); see the text. Vertical blue lines indicate the epoch of the infrared spectrum shown in Figure 6, as well as a second spectral epoch discussed in the text.

From some of these same Spitzer data, Sharma et al. (2012) found [S3.6] or IRAC1 $=14.40 \mathrm{mag}$ and [S4.5] or IRAC2 $=12.89$ mag using a similar 3". 6 radial aperture to us but a smaller 3."6-8". 4 sky annulus. These values are consistent with our results for the first observation epoch, in 2004. We also note that IRSA's SEIP catalog ${ }^{13}$ contains a source SSTSL2 J005220.20+563403.8 with IRAC1 $=14.57 \mathrm{mag}$ and IRAC2 $=12.80 \mathrm{mag}$, adopting the 3 !" 8 aperture values and applying the flux-to-magnitude conversions above, again consistent with our measurements for the 2004 epoch, within the reported errors.

Finally, we note that there are also Spitzer/MIPS observations of the region available in the $24 \mu \mathrm{m}$ band. These MIPS1 data were taken in 2006 February, which is between the two Spitzer/IRAC epochs. It would be valuable to be able to compare the 2006 MIPS1 photometry to the 2010 W4 photometry, for reasons that will become apparent below. Unfortunately, however, the MIPS1 imaging results in only flux upper limits for PGIR 20dci.

\footnotetext{
${ }^{13}$ http://irsa.ipac.caltech.edu/data/SPITZER/Enhanced/SEIP/overview.html
}

\subsubsection{Mid-infrared AllWISE}

WISE images (Cutri et al. 2012) at the comparable bands WISE1 or $W 1(3.4 \mu \mathrm{m})$ and WISE2 or $W 2(4.6 \mu \mathrm{m})$ have lower spatial resolution compared to Spitzer images, but they do show a source with more distinct and point-like appearance in the unWISE image processing (Lang 2014). The AllWISE catalog (Cutri et al. 2012) records a source WISEA J005220.41 +563403.3 with reported photometry given in Table 1 . We took the AllWISE profile fitting magnitudes and errors, as quoted. The measurements are rated as "AAAA" in photometric quality, meaning $\mathrm{S} / \mathrm{N}>10$ in each of the four bands, and they are unsaturated.

\subsubsection{Mid-infrared Colors}

The available colors of the PGIR 20dci progenitor are IRAC1-IRAC2 $=1.60$ and IRAC2-IRAC3 $=1.09 \mathrm{mag}$ from Spitzer and $W 1-W 2=1.12, W 2-W 3=5.82$, and $W 3-W 4=$ 2.76 mag from AllWISE.

These colors place the object firmly within the Class I category of young stellar objects, meaning a steeply rising spectral energy distribution that is best explained by the presence of a massive 
Table 1

Spitzer, WISE, and NEOWISE Photometry ${ }^{\mathrm{a}}$ for PGIR 20dci

\begin{tabular}{|c|c|c|c|c|c|c|c|c|c|c|c|c|c|c|c|c|}
\hline MJD & $\begin{array}{l}\text { WISE1 } \\
3.4 \mu \mathrm{m}\end{array}$ & err & $\begin{array}{l}\text { IRAC1 } \\
3.6 \mu \mathrm{m}\end{array}$ & err & $\begin{array}{l}\text { IRAC2 } \\
4.5 \mu \mathrm{m}\end{array}$ & err & $\begin{array}{l}\text { WISE2 } \\
4.6 \mu \mathrm{m}\end{array}$ & err & $\begin{array}{l}\text { IRAC3 } \\
5.8 \mu \mathrm{m}\end{array}$ & err & $\begin{array}{l}\text { IRAC4 } \\
8.0 \mu \mathrm{m}\end{array}$ & err & $\begin{array}{l}\text { WISE3 } \\
12 \mu \mathrm{m}\end{array}$ & err & $\begin{array}{l}\text { WISE4 } \\
22 \mu \mathrm{m}\end{array}$ & err \\
\hline 53214.65 & (15.01) & $\ldots$ & 14.49 & 0.17 & 12.86 & 0.07 & (12.61) & $\cdots$ & 11.77 & 0.30 & 11.56 & 1.60 & $\cdots$ & $\ldots$ & $\cdots$ & $\cdots$ \\
\hline 55077.57 & (14.49) & $\cdots$ & 13.99 & 0.12 & 12.42 & 0.07 & (12.18) & $\cdots$ & $\ldots$ & $\cdots$ & $\cdots$ & $\cdots$ & $\cdots$ & $\cdots$ & $\cdots$ & $\cdots$ \\
\hline 55080.44 & $(14.45)$ & $\cdots$ & 13.96 & 0.11 & 12.43 & 0.07 & (12.19) & $\cdots$ & $\cdots$ & $\cdots$ & $\cdots$ & $\cdots$ & $\cdots$ & $\cdots$ & $\cdots$ & $\cdots$ \\
\hline (AllWISE) & 12.51 & 0.08 & $\cdots$ & $\cdots$ & $\cdots$ & $\cdots$ & 11.40 & 0.05 & $\cdots$ & $\ldots$ & $\ldots$ & $\ldots$ & 6.11 & 0.04 & 3.36 & 0.07 \\
\hline 55222.15 & 12.63 & 0.25 & $\cdots$ & $\cdots$ & $\cdots$ & $\cdots$ & 11.41 & 0.09 & $\cdots$ & $\cdots$ & $\cdots$ & $\cdots$ & 6.04 & 0.22 & 3.27 & 0.32 \\
\hline 55409.82 & 12.63 & 0.25 & $\ldots$ & $\ldots$ & $\ldots$ & $\ldots$ & 11.41 & 0.09 & $\ldots$ & $\ldots$ & $\ldots$ & $\ldots$ & 6.13 & 0.22 & 3.37 & 0.32 \\
\hline 55586.45 & 12.62 & 0.25 & $\cdots$ & $\cdots$ & $\cdots$ & $\cdots$ & 11.43 & 0.09 & $\cdots$ & $\cdots$ & $\cdots$ & $\cdots$ & $\cdots$ & $\cdots$ & $\ldots$ & $\ldots$ \\
\hline 56684.21 & 12.34 & 0.15 & $\cdots$ & $\ldots$ & $\ldots$ & $\ldots$ & 11.14 & 0.10 & $\ldots$ & $\ldots$ & $\ldots$ & $\ldots$ & $\cdots$ & $\cdots$ & $\cdots$ & $\ldots$ \\
\hline 56873.87 & 12.56 & 0.22 & $\ldots$ & $\ldots$ & $\ldots$ & $\ldots$ & 11.14 & 0.03 & $\ldots$ & $\ldots$ & $\ldots$ & $\ldots$ & $\ldots$ & $\ldots$ & $\ldots$ & $\ldots$ \\
\hline 57045.96 & 12.40 & 0.13 & $\cdots$ & $\cdots$ & $\cdots$ & $\cdots$ & 11.07 & 0.10 & $\cdots$ & $\cdots$ & $\cdots$ & $\cdots$ & $\cdots$ & $\cdots$ & $\cdots$ & $\cdots$ \\
\hline 57235.95 & 12.43 & 0.21 & $\cdots$ & $\cdots$ & $\cdots$ & $\cdots$ & 11.12 & 0.07 & $\cdots$ & $\cdots$ & $\cdots$ & $\cdots$ & $\cdots$ & $\cdots$ & $\cdots$ & $\cdots$ \\
\hline 57408.47 & 12.26 & 0.26 & $\ldots$ & $\ldots$ & $\ldots$ & $\ldots$ & 11.00 & 0.01 & $\ldots$ & $\ldots$ & $\ldots$ & $\ldots$ & $\ldots$ & $\ldots$ & $\ldots$ & $\ldots$ \\
\hline 57602.68 & 12.31 & 0.26 & $\cdots$ & $\cdots$ & $\cdots$ & $\cdots$ & 10.97 & 0.07 & $\cdots$ & $\cdots$ & $\cdots$ & $\cdots$ & $\cdots$ & $\cdots$ & $\cdots$ & $\cdots$ \\
\hline 57769.55 & 12.20 & 0.20 & $\cdots$ & $\cdots$ & $\cdots$ & $\cdots$ & 10.89 & 0.05 & $\cdots$ & $\cdots$ & $\cdots$ & $\cdots$ & $\cdots$ & $\cdots$ & $\cdots$ & $\cdots$ \\
\hline 57966.88 & 12.32 & 0.17 & $\cdots$ & $\cdots$ & $\cdots$ & $\cdots$ & 10.85 & 0.06 & $\cdots$ & $\cdots$ & $\cdots$ & $\cdots$ & $\cdots$ & $\cdots$ & $\cdots$ & $\cdots$ \\
\hline 58129.81 & 12.02 & 0.17 & $\cdots$ & $\cdots$ & $\cdots$ & $\cdots$ & 10.68 & 0.06 & $\cdots$ & $\cdots$ & $\cdots$ & $\cdots$ & $\cdots$ & $\cdots$ & $\cdots$ & $\cdots$ \\
\hline 58331.39 & 12.09 & 0.18 & $\cdots$ & $\cdots$ & $\cdots$ & $\cdots$ & 10.62 & 0.05 & $\cdots$ & $\cdots$ & $\cdots$ & $\cdots$ & $\cdots$ & $\cdots$ & $\cdots$ & $\cdots$ \\
\hline 58491.38 & 11.83 & 0.18 & $\ldots$ & $\ldots$ & $\ldots$ & $\ldots$ & 10.50 & 0.03 & $\cdots$ & $\cdots$ & $\cdots$ & $\cdots$ & $\cdots$ & $\cdots$ & $\cdots$ & $\cdots$ \\
\hline 58698.13 & 11.17 & 0.10 & $\cdots$ & $\ldots$ & $\cdots$ & $\cdots$ & 9.70 & 0.04 & $\cdots$ & $\cdots$ & $\cdots$ & $\cdots$ & $\cdots$ & $\cdots$ & $\cdots$ & $\cdots$ \\
\hline 58858.12 & 10.35 & 0.09 & $\cdots$ & $\cdots$ & $\cdots$ & $\cdots$ & 8.97 & 0.03 & $\cdots$ & $\cdots$ & $\cdots$ & $\cdots$ & $\cdots$ & $\cdots$ & $\cdots$ & $\cdots$ \\
\hline 59062.32 & 10.25 & 0.10 & $\cdots$ & $\cdots$ & $\cdots$ & $\cdots$ & 8.89 & 0.03 & $\cdots$ & $\cdots$ & $\cdots$ & $\cdots$ & $\cdots$ & $\cdots$ & $\cdots$ & $\cdots$ \\
\hline 59222.09 & 10.20 & 0.08 & $\cdots$ & $\cdots$ & $\cdots$ & $\cdots$ & 8.93 & 0.03 & $\cdots$ & $\cdots$ & $\cdots$ & $\cdots$ & $\cdots$ & $\cdots$ & $\cdots$ & $\ldots$ \\
\hline
\end{tabular}

Note.

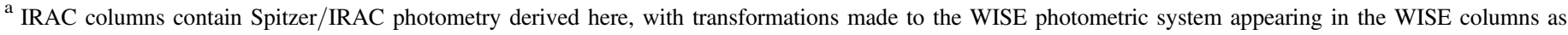

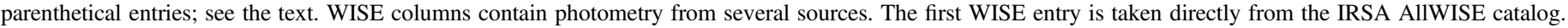

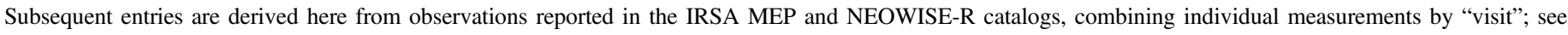
the text.

circumstellar envelope. The colors are too red to be consistent with a geometrically flatter disk, as is the case for Class II sources. PGIR 20dci is, however, even redder than most Class I sources, which indicates substantial foreground extinction.

\subsection{WISE+NEOWISE Light Curve}

In this section, we present mid-infrared data that support the PGIR detection of a significant brightening of the source.

We collected photometry from the WISE Multi-Epoch and NEOWISE (Cutri et al. 2015) mission archives via IRSA, ${ }^{14}$ rejecting measurements with qi_fact $=0$ or qual_frame $=0$. We checked the WISE/NEOWISE photometry flags, ${ }^{15}$ finding no entries in the $w[12] c c \_m a p \_s t r$ (contamination and confusion) column, and only nonevent entries in the na (deblending) column. The $n b$ (multiple PSF) column indicates that a single profile is used in $>80 \%$ of the measurements; only $<20 \%$ of the measurements (all toward the fainter end) require a second PSF, perhaps to account for the faint star just to the north of PGIR 20dci (see the Spitzer image panel in Figure 3).

For the WISE Multi-Epoch catalog and the subsequent, ongoing NEOWISE monitoring data, we took the median of all profile fitting magnitude measurements within each spacecraft "visit" covering the position, and we calculated the error as the dispersion among these magnitudes. The median time span per WISE or NEOWISE visit is about 1.25 days. Results are provided in Table 1.

\footnotetext{
${ }^{14}$ https://irsa.ipac.caltech.edu/Missions/wise.html

15 https://wise2.ipac.caltech.edu/docs/release/neowise/expsup/sec2_1a.html
}

As illustrated in Figure 3, the mid-infrared light curve of PGIR 20dci experienced an exponential rise phase over the past few years, which appears to be plateauing during 2020. Considering only the three NEOWISE measurements at $\mathrm{MJD}=58491.8,58698.2$, and 58858.1, before the flattening, the rise rate was $-0.121 \pm 0.015$ mag month ${ }^{-1}$ or -1.40 mag $\mathrm{yr}^{-1}$ during 2019-2020. We note that this is identical, within the errors, to the slope derived for the $J$-band light curve $\left(-0.116 \pm 0.011 \mathrm{mag}\right.$ month $^{-1}$; see discussion above regarding Figure 2). Before this steep recent rise, there was a much shallower but steady upward trend in the WISE/NEOWISE light curve between 2010 and 2019, with a rise slope of only

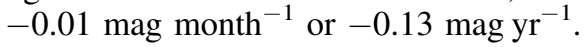

\subsection{Overall Mid-infrared Light and Color Evolution}

As discussed in Section 3.2.2, prior to the 2010 WISE mission measurements, there are data available in 2009 and 2004 from Spitzer. A shallow rise is also seen in these data, though there are a limited number of data points. The steeper jump that is implied between the Spitzer and WISE epochs occurs in a fairly narrowly confined time frame, within 2009 September 6 (Spitzer) and 2010 January 26 (WISE), and warrants critical investigation.

The two shortest-wavelength Spitzer and WISE filters are similar, and Jarrett et al. (2011) find agreement in large-scale photometry comparisons to within 2\%-3\%. However, PGIR 20dci is an extremely red source, beyond the range of colors available to Jarrett et al. (2011), and thus we need to consider potential color terms between the earlier Spitzer and later WISE photometry. Similar considerations were also made for the 

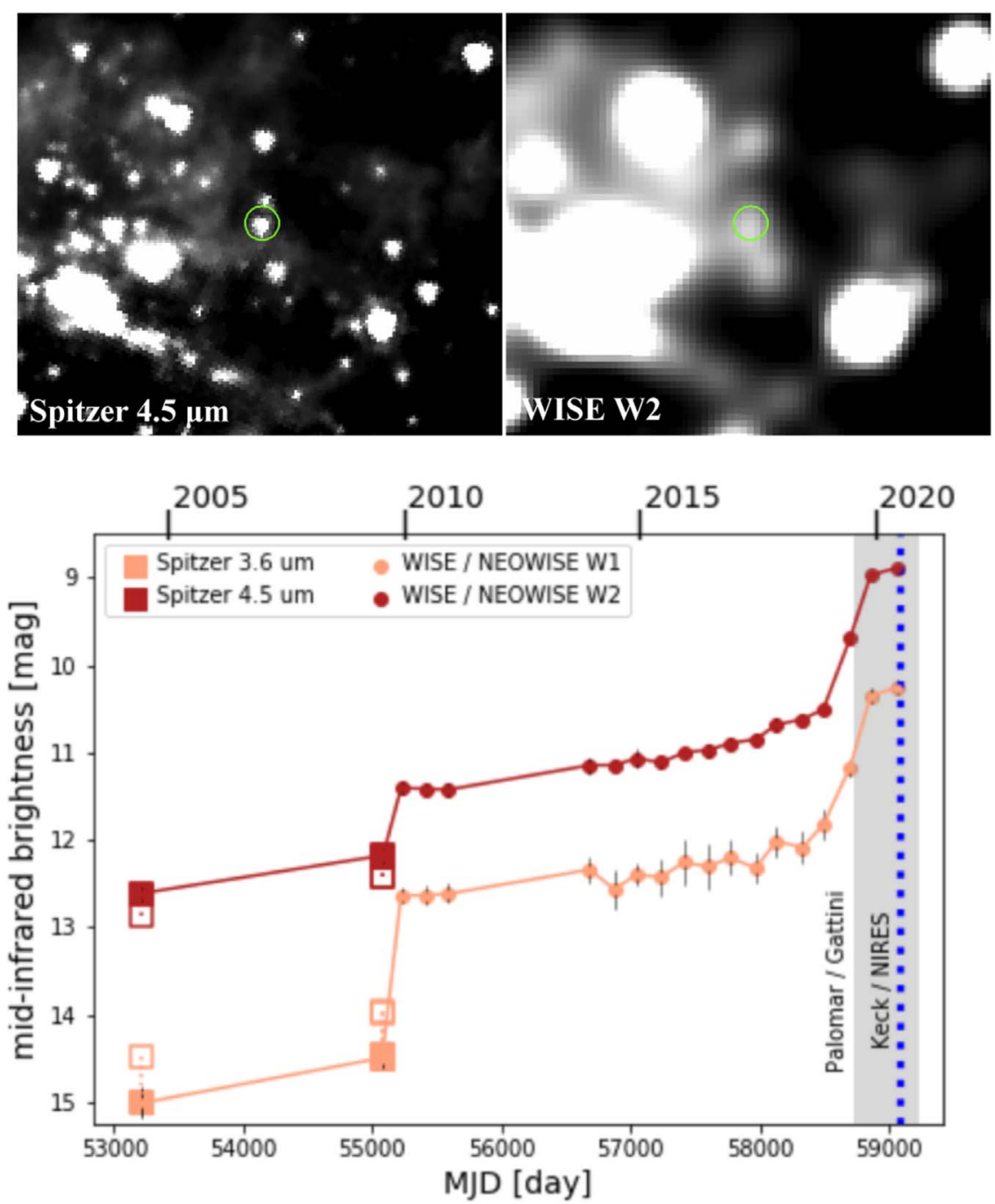

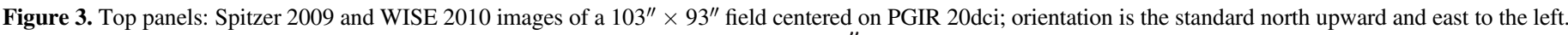

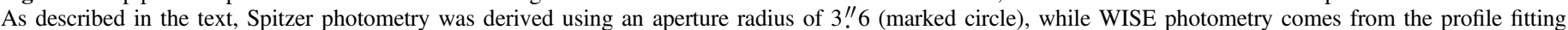

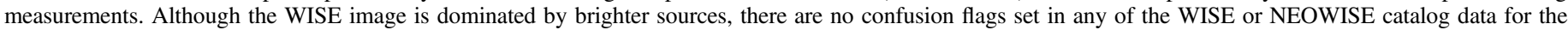

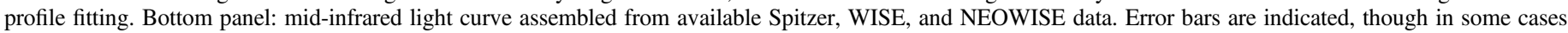

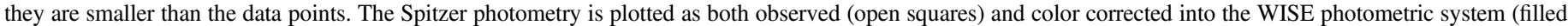

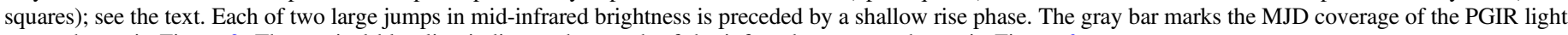
curve shown in Figure 2. The vertical blue line indicates the epoch of the infrared spectrum shown in Figure 6.

light-curve analysis of Gaia 17bpi (Hillenbrand et al. 2018) and PTF 14jg (Hillenbrand et al. 2019), where color transformations were derived ${ }^{16}$ and implemented, but not magnitude transformations. Here for PGIR 20dci, we have tried to assess more carefully the impact of color terms on the light curve, by looking at a large sample of young stellar objects observed with both Spitzer and WISE. While there is a narrow correlation in the magnitudes themselves, when considering the IRAC $1-W 1$ and IRAC2 $-W 2$ transformations as a function of IRAC1 -IRAC2 color, the comparison data show a fanning out toward redder IRAC1-IRAC2 color, which introduces significant uncertainty. Identical issues were investigated by Antoniucci et al. (2014, see their Section 2.1.1), and we have adopted their corrections. $^{17}$ For the IRAC1-IRAC2 color of PGIR 20dci, this amounts to shifts of approximately $0.5 \mathrm{mag}$ fainter in going from IRAC1 to $W 1$ and -0.25 mag brighter in going

\footnotetext{
$16(W 1-W 2)_{\text {corr }}=1.62 \times($ IRAC1 - IRAC2 $)-0.04$ mag, with rms $=0.24$ mag $17 W 1_{\text {corr }}=\operatorname{IRAC} 1-0.27489 \times(\operatorname{IRAC} 2-\operatorname{IRAC} 1)+0.07146$ and $W 2_{\text {corr }}=$ IRAC2 $+0.1422 \times($ IRAC2 - IRAC1 $)-0.01855$.
}

from IRAC2 to $W 2$. Figure 3 illustrates both the observed and the WISE-corrected Spitzer magnitudes, which also appear in the WISE columns of Table 1.

In order to negate the conclusion of a large jump in brightness between the last Spitzer epoch and the first WISE epoch, the color corrections to the photometry would have to be much larger than currently supposed (which is certainly possible), but also in the opposite direction for the Spitzer IRAC1 channel. We also note that, even among the very red sources in this spatial region, PGIR 20dci is an outlier in direct comparisons of Spitzer versus AllWISE photometry, lending support to the reality of the apparent brightness jump.

We can further scrutinize the WISE/NEOWISE photometry by considering reported aperture magnitudes. The aperture photometry exhibits the expected trends with aperture size, with the 5."5 aperture undersized and producing magnitude overestimates, and other apertures oversized and thus having magnitudes that well underestimate the profile fitting magnitudes. Even if the undersized 5!"5 aperture photometry were 
used, the brightness jump between the Spitzer 2009 and WISE 2010 measurements would remain, albeit reduced by about 0.25 and $0.75 \mathrm{mag}$ in $W 1$ and $W 2$, respectively. We note that the $w$ [12]flg_l (5!"5 aperture confusion) column indicates that about $8 \%$ of the aperture photometry measurements have some identified source of confusion; this is not significant for the wlmpro magnitude measurements that we have adopted, however. Overall, the profile fitting photometry is more robust to bad pixels, cosmic rays, and the need to establish aperture corrections.

Finally, we can consider the evidence for color changes as the source has brightened in the mid-infrared. The recent steep brightening of PGIR 20dci has been colorless, within the errors. The median color over the 2014-2020 NEOWISE epochs is consistent with a constant value of $W 1-W 2=1.40$ mag and $\mathrm{rms}=0.21 \mathrm{mag}$. Earlier, in 2010, however, the WISE colors were $W 1-W 2 \approx 1.23$ mag for each of the three epochs, so marginally bluer in the period just after the 2009-2010 jump in brightness. The even earlier 2009 and 2004 Spitzer colors are both IRAC1-IRAC2 $\approx 1.60$, which, after applying the Spitzerto-WISE color transformation quoted above, results in a corresponding $W 1-W 2=2.55 \mathrm{mag}$. Applying the Antoniucci et al. (2014) magnitude corrections detailed above results in fairly similar colors of 2.40, 2.31, and 2.26 mag for the three Spitzer epochs.

The above suggests that the color of PGIR 20dci became bluer by about 1 mag in $W 1-W 2$ as it brightened during the late 2009 and very early 2010 period, before reddening by a small amount $(\approx 0.2 \mathrm{mag})$ and then continuing to brighten colorlessly. It is hard to know how to interpret this overall behavior. If driven by an accretion event, one would expect some blueing behavior. At the same time, there may be extinction clearing going on, which would also imply a color change to the blue. The neutral color behavior that is observed over the past $5 \mathrm{yr}$ as PGIR 20dci has brightened seems anomalous with respect to these expectations.

In summary, the mid-infrared light curve of PGIR 20dci shows that the source has undergone a long-timescale, two-step rise from a faint state to a bright state over the past $\sim 15 \mathrm{yr}$. During the period 2004-2019, the source increased its midinfrared brightness by $>5 \mathrm{mag}$ in total. There was a shallow brightening from 2004 to 2009, a first significant jump in brightness during a 4-month time frame in late 2009 or early 2010, a second shallow rise phase, and a more recent second significant brightness jump beginning in 2019 and plateauing in 2020 .

\section{Follow-up Observations and Data Analysis}

In this section, we describe the imaging and spectroscopy that we collected on PGIR 20dci.

\subsection{Imaging}

\subsubsection{Data Acquisition}

PGIR 20dci was flagged for follow-up with the Palomar 60" telescope and SEDM (Spectral Energy Distribution Machine; Blagorodnova et al. 2018) in its imaging mode. A sequence in the gri filters was acquired on 2020 August 14 (UT) and reduced using the photometric reduction pipeline.

On 2020 August 28, we obtained near-infrared imaging of PGIR 20dci using the Wide Field Infrared Camera (WIRC; Wilson et al. 2003) on the Palomar 200-inch telescope. The

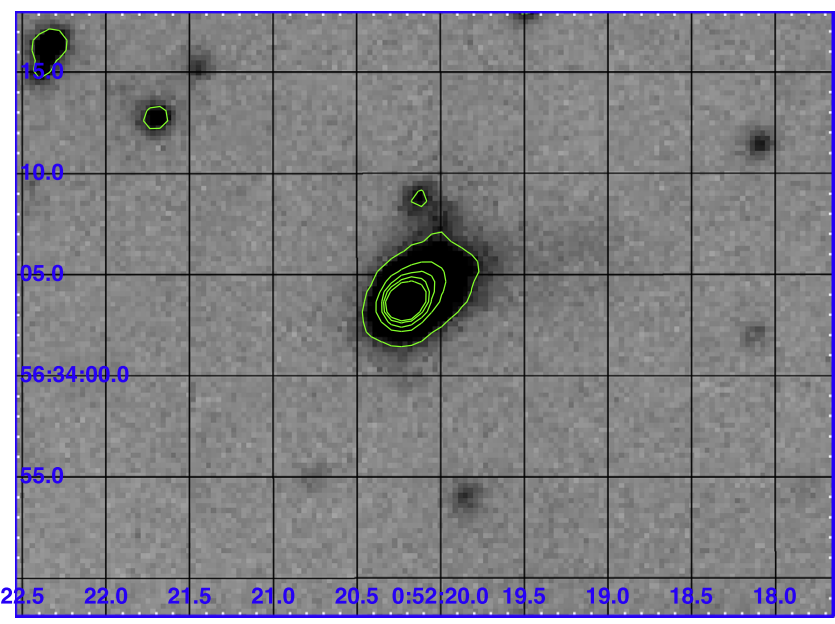

Figure 4. Approximately $40^{\prime \prime} \times 30^{\prime \prime}$ cutout from the Palomar/WIRC $K_{s}$ $(2.2 \mu \mathrm{m})$ image of Figure 1, centered near PGIR 20dci. Grid spacing is 5 time seconds in R.A., and $5^{\prime \prime}$ in decl. The extended structure is oriented southeast to northwest, with concentrated flux toward the southeast and increasingly diffuse emission toward the northwest, including beyond the lowest contour. The extent of the scattered light within the contours is $\sim 14,000$ au.

data were acquired as a series of dithered exposures in $J, H$, and $K_{s}$ bands for a total exposure time of 495, 330, and $300 \mathrm{~s}$, respectively. The data were reduced, stacked, and photometrically calibrated using the pipeline described in De et al. (2020a).

\subsubsection{Findings}

No counterpart to PGIR 20dci was detected in $g$ and $r$ bands, with an approximate upper limit of $>21$ mag. However, we detect a faint extended source in $i$ band, coincident with the bright nebula detected in the near-infrared image. The final $K_{s}$-band image was shown in Figure 1 . A close-up on PGIR 20dci appears in Figure 4, which highlights the extended cometary structure of the source in the near-infrared. Figure 5 shows the $J H K$ color composite image.

Taken together, the optical and infrared imaging suggests an extremely red source, as well as a bright, extended, scattered light component associated with the brightening of PGIR 20dci. The angular size of the extended structure corresponds to a physical size of $\sim 14,000$ au.

In order to encompass the non-point-source, extended nature of PGIR 20dci, we measured photometry from the Palomar/ WIRC images using a fairly large aperture radius of 9 pixels (2! 25), whereas a 5-pixel (1".25) radius would suffice for point sources. Using the smaller radius would decrease the brightness of PGIR 20dci by about $0.1 \mathrm{mag}$. We calibrated by matching to 110 sources in common between our final images and the 2MASS catalog. We find $J=14.83, H=13.08$, and $K_{s}=11.77$ mag in 2020 September. The near-infrared colors of PGIR 20dci in outburst are thus $J-H=1.75 \mathrm{mag}$ and $H-K_{s}=1.31$ mag. This location in a $J-H$ versus $H-K$ diagram is consistent with that of a young stellar object that is highly reddened.

\subsection{Spectroscopy}

\subsubsection{Data Acquisition}

On 2020 August 13, PGIR 20dci was observed with Keck II and Near Infrared Echellette Spectrometer (NIRES), which is 


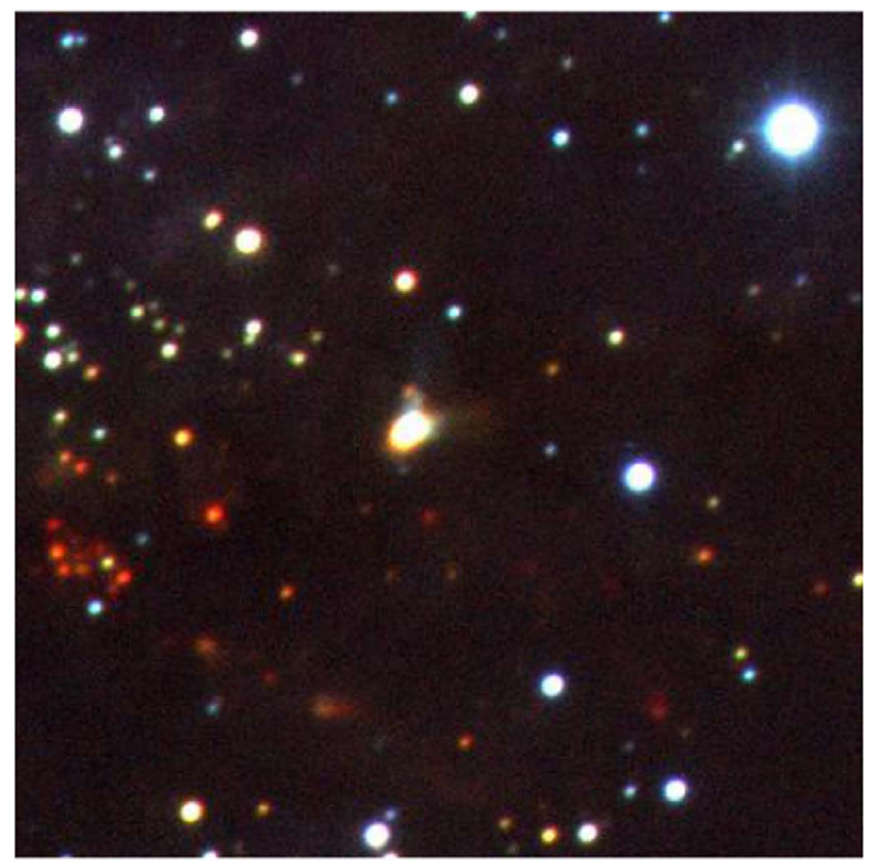

Figure 5. Color rendering of the Palomar/WIRC $J H K$ data, in standard orientation of north upward and east to the left. PGIR 20dci in outburst is the extended source near center frame. Faintly visible are extended "tails" of emission to the northwest, which may indicate the orientation of the outflow axis.

part of the family of similar instruments described in Wilson et al. (2004). The detector records a prism-dispersed simultaneous $Y J H K$ spectrum at resolution $R \approx 2700$. Four spectra were acquired using a 6" ABBA nodding pattern, with integration time of $90 \mathrm{~s}$ at each position. The data were reduced using a modified version of spextool (Cushing et al. 2004) and flux-calibrated with the telluric A0V standard star HIP 6002 using the xtellcor code (Vacca et al. 2003). Figure 6 shows the final extracted and combined spectrum. Due to the very red nature of the source, there is little signal at the shorter wavelengths, and thus we show only the $J H K$ spectral region.

On 2020 September 4, a second spectrum was obtained at $R \approx 2700$ with the Palomar 200" and the TripleSpec (Herter et al. 2008) near-infrared spectrograph. Although with a lower $\mathrm{S} / \mathrm{N}$, and not shown as a result, the spectrum is broadly the same in its shape and presence of strong spectral features as the Keck/NIRES spectrum from a month earlier. However, the flux level is brighter. Considering Figure 2, the flux at this point in time could have been several tenths of a magnitude brighter, though the error on this estimate is large. A more likely explanation is that the TripleSpec slit (1"!0) is wider than the NIRES slit (0". 55), and thus the spectrum would include more of the bright extended structure of the source (see Figure 4). Normalizing the two at $K$ band, the $K$-band slope is identical to that shown in Figure 6, but there is a sharper break just before the $\mathrm{CO}$ region, resulting in even deeper $\mathrm{CO}$ absorption. The $\mathrm{H}$ band region is then slightly brighter than the spectrum shown in Figure 6 . This could mean that the source became slightly bluer while temporarily brighter at this epoch. However, we ultimately attribute the difference to the possibility that the two spectra may have included different parts of the extended nebula.

\subsubsection{Findings}

Focusing on the Keck/NIRES spectrum (Figure 6), PGIR $20 \mathrm{dci}$ is highly reddened, consistent with the extremely red infrared colors measured by WIRC and WISE. The comparison objects $^{18}$ in Figure 6 are less reddened than PGIR 20dci. We can estimate the relative reddening, and thus the extinction, by applying a standard extinction law to find the best spectral match to the V1057 spectrum. We find a differential extinction $\Delta A_{V}=16 \mathrm{mag}$, which can be added to a baseline $A_{V}=4 \mathrm{mag}$ for V1057 (Connelley \& Reipurth 2018). The total extinction toward PGIR 20dci is thus estimated to be $A_{V} \approx 20 \mathrm{mag}$.

Spectroscopically, the most salient feature of PGIR 20dci is the strong CO absorption. The source has both the $\Delta \nu=2 \mathrm{CO}$ bands in the $K$ band and the $\Delta \nu=3$ bands $\mathrm{CO}$ in the $H$ band. There is also strong $\mathrm{H}_{2} \mathrm{O}$ in $J$ band and $H$ band, and probably in $K$ band as well, and likely $\mathrm{OH}$ in $H$ band. Less obvious is the expected $\mathrm{TiO}$ and $\mathrm{VO}$ absorption, though it may be weakly present. PGIR 20dci shares with Gaia 17bpi and V1057 Cyg in having clear $J$-band $\mathrm{H}_{2} \mathrm{O}$ absorption and $\mathrm{H}$ - and $K$-band $\mathrm{CO}$ absorption. The $\mathrm{H}_{2} \mathrm{O}$ is weaker in PGIR 20dci, while the $\mathrm{CO}$ is stronger. We note that the CO in V1057 Cyg is atypically weak for FU Ori stars (Connelley \& Reipurth 2018).

In terms of atomic lines, weak $\mathrm{Na} \mathrm{I}, \mathrm{Mg}$ I, $\mathrm{Al} \mathrm{I}, \mathrm{Si}$ I, and $\mathrm{Ca}$ I absorptions are all present. There is also weak $\mathrm{HI} \mathrm{Pa} \beta$ absorption at $1.28 \mu \mathrm{m}$. The metal line strengths are similar to those seen in the comparison object Gaia 17bpi and somewhat stronger than in V1057 Cyg. PGIR 20dci and Gaia 17bpi also have comparable $\mathrm{Pa} \beta$, which is likely blueshifted and formed in a wind.

Notably, the He I $\lambda 10830$ line in PGIR 20dci shows a clear P Cygni profile (see inset panel within Figure 6). This is evidence of a strong wind or outflow, with blueshifted absorption in He I $\lambda 10830$ frequently seen in FU Ori stars (Connelley \& Reipurth 2018). We estimate a maximum velocity around $-450 \mathrm{~km} \mathrm{~s}^{-1}$.

In the nomenclature of Connelley \& Reipurth (2018), PGIR 20dci appears to be a bona fide FU Ori star. In addition to having had its eruption observed, it satisfies all of the spectroscopic criteria: $\mathrm{CO}$ absorption, $\mathrm{H}_{2} \mathrm{O}$ absorption, $\mathrm{VO}$ or $\mathrm{TiO}$ absorption (weakly), $\mathrm{Pa} \beta$ absorption, lack of emission lines, weak metal absorption, and He I $\lambda 10830$ absorption. The hydrogen and helium lines likely have some outflow aspect to their absorption. The metal lines originate in the source photosphere, however, and combined with the molecular line absorption, they indicate a mixed-temperature spectrum.

\section{Discussion}

The dramatic brightening behavior of PGIR 20dci is detected most obviously in the mid-infrared (Figure 3 ). The combined Spitzer, WISE, and NEOWISE data provide a long-duration light curve. As the source has brightened, it has become detectable in the near-infrared as well (Figure 2). In the PGIR data, which are higher cadence, the brightness of PGIR 20dci is currently near the survey limit owing to the high background in this field, and the light curve is thus somewhat noisy, though well sampled. Over only the past few months, PGIR 20dci has

\footnotetext{
$\overline{18}$ Gaia 17bpi is a recent FU Ori outburst occurring in 2017 (Hillenbrand et al. 2018), which is still near its peak brightness. V1057 Cyg is one of the originally defined FU Ori stars, estimated to have outburst in 1970 (Herbig 1977), and although it has faded considerably, the spectrum maintains the general features of FU Ori stars (e.g., Connelley \& Reipurth 2018).
} 


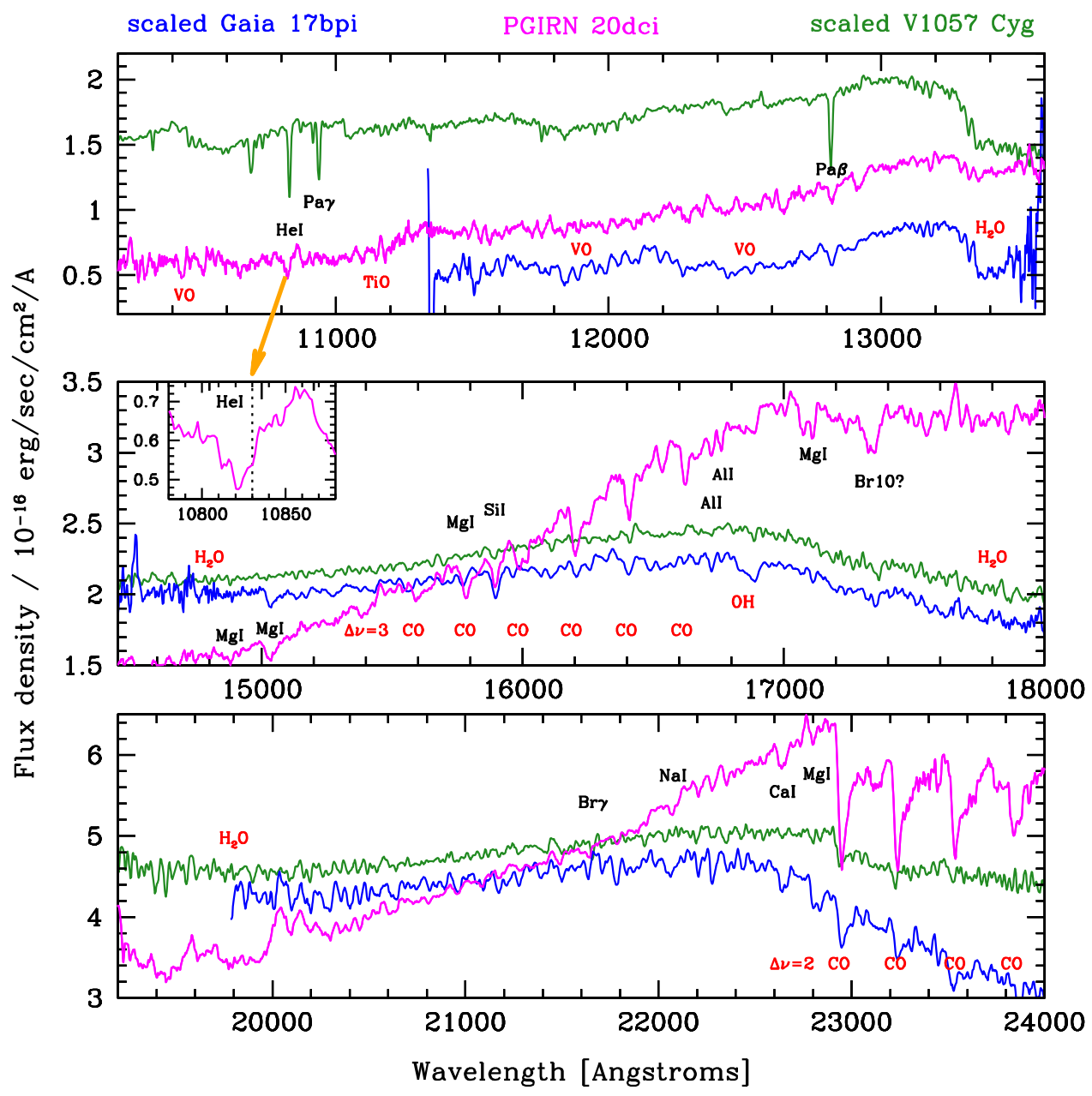

Figure 6. Comparison of PGIR 20dci (magenta) with the FU Ori stars V1057 Cyg (green; from Connelley \& Reipurth 2018) and Gaia 17bpi (blue; from Hillenbrand et al. 2018). For purposes of plotting, the comparison objects have been scaled and shifted, with the corrections differing among the different panels. The spectral match is apparent in molecular ${ }^{12} \mathrm{CO}, \mathrm{H}_{2} \mathrm{O}$, and $\mathrm{OH}$, and possibly weak TiO and VO. Also present are atomic lines of $\mathrm{H} \mathrm{I} \mathrm{Pa} \beta$ (and possibly Br10), $\mathrm{He}$ I $\lambda 10830$, and metals including Na I, Mg I, Al I, Si I, and Ca I. While the hydrogen and helium lines likely have some outflow aspect to their absorption, the metal lines originate in the photosphere and, combined with the molecular line absorption, indicate a mixed-temperature spectrum.

appeared near the faint limit in the ZTF alert stream, sampling the red optical. Given the evidence for a current (second) plateau phase, the source may not become amenable to study in the optical, however.

A two-step rise is indicated by the mid-infrared light curve, each step being preceded by a shallow rise phase. The earliest available data for PGIR 20dci show a shallow brightening between 2004 and 2009. Then, PGIR 20dci exhibited a rapid $\left(-0.25 \mathrm{mag}\right.$ month $\left.^{-1}\right)$ blue brightening by $1.8 \mathrm{mag}$ in $W 1$ and 0.8 mag in $W 2$ between 2009 September 6 and 2010 January 26. This was followed by a shallower-slope (-0.01 mag month ${ }^{-1}$ ), long-term colorless brightening over 2010-2018, amounting to only another $0.5 \mathrm{mag}$ in each of $W 1$ and $W 2$. The slope of the brightening increased during the latter part of 2018 and switched in 2019 to a much steeper rise rate $(-0.12 \mathrm{mag}$ month ${ }^{-1}$ ). At this point, the source became detectable by PGIR in the near-infrared. PGIR $(1.2 \mu \mathrm{m})$ and NEOWISE (3.4 and $4.6 \mu \mathrm{m})$ both record the same rise slope in this time frame, and both show a transition to a second plateau during 2020. The total brightening of PGIR 20dci over the past $16-25 \mathrm{yr}$ is $\sim 5-5.5$ mag.

Putting these light-curve results into context, the recent rise slope compares well to the typical rise rates that can be derived for the optical light curves of historically documented FU Ori outbursts. These have a wide range but are most frequently about -0.05 to $-0.2 \mathrm{mag}$ month $^{-1}$ (L. A. Hillenbrand 2021, in preparation). Regarding the two-step rise in the mid-infrared light curve, a similar phenomenon has been recorded on one other source: the confirmed FU Ori source Gaia 17bpi (Hillenbrand et al. 2018). However, in that object, the total duration of the first plateau phase was shorter, only 1-2 yr, instead of the 5-10 yr measured here for PGIR 20dci.

We can estimate a source luminosity near the outburst peak based on the brightness level of the apparent plateau in the light curve $(J \approx 14.6 \mathrm{mag}$ from PGIR and $r \approx 20.5 \mathrm{mag}$ from ZTF) and the adopted distance $d=2.81 \mathrm{kpc}$. The bolometric correction is of course unknown, but adopting values appropriate for a mid-F-type star leads to a luminosity estimate of $10-12 L_{\odot}$, with perhaps a factor of 3 uncertainty if the bolometric correction is $\pm 1 \mathrm{mag}$. This is on the far low side of FU Ori outburst peak luminosities but notably does not include the long wavelength contribution, which is currently unmeasured. Just Gaia 17bpi, the only other source known to have exhibited a two-step mid-infrared brightening, like PGIR 20dci has, and HOPS 383, an embedded young star outburster whose status as an FU Ori-type outburst is unclear, have luminosities this low. 
Concluding our discussion, we note that the best-studied FU Ori stars are the optically visible portion of the population, especially those bright enough for high-dispersion spectroscopy. However, a sizable fraction of the known FU Ori stars, about one-third, are seen only at infrared wavelengths owing to high extinction (e.g., the $A_{V}=20-50 \mathrm{mag}$ population in Connelley \& Reipurth 2018). Indeed, theoretical predictions are that earlier-stage young stellar objects experience a higher rate of FU Ori-type outbursts (Bae et al. 2014). Most of the known optically invisible group have been designated as FU Ori objects through source brightening at $K$ band that was noticed only post facto, rather than while it was actually happening, along with spectroscopic confirmation. Embedded FU Ori sources thus generally do not have quiescence-to-rise light curves available.

Because it meets all applicable criteria for the FU Ori designation, notably the spectral absorption signatures, a lack of emission lines other than P Cygni structure, and the total lightcurve amplitude, PGIR 20dci can be added to the list of optically invisible FU Ori candidates. Unlike the others, however, this source has had its photometric rise captured through wide-field continuous photometric monitoring programs.

\section{Summary}

We have discovered an outbursting young stellar object first detected at near-infrared wavelengths, but also seen in long-duration mid-infrared time series data. PGIR 20dci is associated with the NGC 281-W molecular core, a known area of recent and ongoing dense star formation activity. Its salient features are as follows:

1. A Class I spectral energy distribution.

2. Foreground interstellar, molecular core, and circumstellar extinction amounting to $A_{V} \approx 20 \mathrm{mag}$.

3. A photometric rise that was detected in the PGIR timedomain survey and emphasized by comparing the recent $J$-band photometry with the previous lack of a $J$-band detection at the same position, implying a total nearinfrared brightness increase of $>5 \mathrm{mag}$.

4. Extended nebulosity in our new $K_{s}$-band imaging, which was not present in 1990s images at the same wavelength, with a cometary-type structure $\sim 14,000$ au in size.

5. A measured 10-15 yr photometric brightening trend exhibited in 3-5 $\mu \mathrm{m}$ Spitzer + WISE + NEOWISE data, accumulating a total mid-infrared brightness increase of $>5$ mag.

6. Steepened mid-infrared brightening during 2019 that was coincident with source detection at $J$ band.

7. Consistent rise rates during 2018-2020 in the midinfrared NEOWISE light curve and during 2019-2020 in the near-infrared PGIR light curve, amounting to -0.12 mag month ${ }^{-1}$.

8. A flattening of the light curve from its rise phase to a plateau in 2020. The peak luminosity is estimated at a modest $10-12 L_{\odot}$, though this is likely a lower limit.

9. A near-infrared absorption-line spectrum showing hydrogen line, metal line, and molecular band absorption, indicative of a "mixed-temperature" spectrum.

10. Outflow as evidenced by an He I $\lambda 10830$ profile, with blueshifted absorption seen to about $-450 \mathrm{~km} \mathrm{~s}^{-1}$.
We conclude that PGIR 20dci is a bona fide FU Ori star. The object warrants further study, especially at high spectral dispersion, as its outburst continues to develop.

Palomar Gattini-IR (PGIR) is generously funded by Caltech, Australian National University, the Mt. Cuba Foundation, the Heising Simons Foundation, and the Bi- national Science Foundation. PGIR is a collaborative project among Caltech, Australian National University, University of New South Wales, Columbia University, and the Weizmann Institute of Science. M.M.K. acknowledges generous support from the David and Lucille Packard Foundation.

Facilities: Palomar Gattini IR, PO:1.2 m:ZTF, PO:1.5 m: SEDM, Hale:WIRC, Hale:TSPEC, Keck/NIRES, Keck/ DEIMOS, Spitzer, WISE, NEOWISE, IRSA.

\section{ORCID iDs}

Kishalay De (D) https://orcid.org/0000-0002-8989-0542 Matthew Hankins (iD https://orcid.org/0000-0001-9315-8437 Mansi M. Kasliwal (10 https://orcid.org/0000-0002-5619-4938

Luisa M. Rebull (D) https://orcid.org/0000-0001-6381-515X

Roc M. Cutri (i) https://orcid.org/0000-0002-0077-2305

Michael C. B. Ashley (D) https://orcid.org/0000-00031412-2028

Viraj R. Karambelkar (ib https://orcid.org/0000-00032758-159X

T. Travouillon (ib https://orcid.org/0000-0001-9304-6718

\section{References}

Antoniucci, S., Giannini, T., Li Causi, G., et al. 2014, ApJ, 782, 51 Armitage, P. J. 2019, SAAS, 45, 1

Bae, J., Hartmann, L., Zhu, Z., \& Nelson, R. P. 2014, ApJ, 795, 61 Bellm, E. C., Kulkarni, S. R., Graham, M. J., et al. 2019, PASP, 131, 018002 Blagorodnova, N., Neill, J. D., Walters, R., et al. 2018, PASP, 130, 035003 Carpenter, J. M., Snell, R. L., Schloerb, F. P., et al. 1993, ApJ, 407, 657 Cody, A. M., \& Hillenbrand, L. A. 2018, AJ, 156, 71 Connelley, M., \& Reipurth, B. 2020, ATel, 14035, 1

Connelley, M. S., \& Reipurth, B. 2018, ApJ, 861, A145

Costigan, G., Vink, J. S., Scholz, A., et al. 2014, MNRAS, 440, 3444

Cushing, M. C., Vacca, W. D., \& Rayner, J. T. 2004, PASP, 116, 362

Cutri, R. M., Mainzer, A., Conrow, T., et al. 2015, Explanatory Supplement to the NEOWISE Data Release Products, http://wise2.ipac.caltech.edu/docs/ release/neowise/expsup

Cutri, R. M., Skrutskie, M. F., van Dyk, S., et al. 2003, 2MASS All Sky Catalog of Point Sources (Washington, DC: NASA)

Cutri, R. M., Wright, E. L., Conrow, T., et al. 2012, Explanatory Supplement to the WISE All-Sky Data Release Products, https://wise2.ipac.caltech.edu/ docs/release/allwise/expsup/

De, K., Hankins, M. J., Kasliwal, M. M., et al. 2020a, PASP, 132, 025001

De, K., Kasliwal, M. M., Tzanidakis, A., et al. 2020b, ApJ, 905, 58

Elmegreen, B. G., \& Lada, C. J. 1978, ApJ, 219, 467

Fazio, G. G., Hora, J. L., Allen, L. E., et al. 2004, ApJS, 154, 10

Findeisen, K., Cody, A. M., \& Hillenbrand, L. 2015, ApJ, 798, 89

Flewelling, H. A., Magnier, E. A., Chambers, K. C., et al. 2020, ApJS, 251, 7 Gaia Collaboration, Brown, A. G. A., Vallenari, A., et al. 2018, A\&A, 616, A1 Graham, M. J., Kulkarni, S. R., Bellm, E. C., et al. 2019, PASP, 131, 078001 Griffin, M. J., Abergel, A., Abreu, A., et al. 2010, A\&A, 518, L3

Guetter, H. H., \& Turner, D. G. 1997, AJ, 113, 2116

Herbig, G. H. 1977, ApJ, 217, 693

Herter, T. L., Henderson, C. P., Wilson, J. C., et al. 2008, Proc. SPIE, 7014, $70140 \mathrm{X}$

Hillenbrand, L. A., Contreras Peña, C., Morrell, S., et al. 2018, ApJ, 869, 146 Hillenbrand, L. A., Miller, A. A., Carpenter, J. M., et al. 2019, ApJ, 874, 82 Holland, W., MacIntosh, M., Fairley, A., et al. 2006, Proc. SPIE, 6275, 62751E Jarrett, T. H., Cohen, M., Masci, F., et al. 2011, ApJ, 735, 112 Lang, D. 2014, AJ, 147, 108

Law, N. M., Kulkarni, S. R., Dekany, R. G., et al. 2009, PASP, 121, 1395 Lee, Y., \& Jung, J.-H. 2003, NewA, 8, 191 
Mainzer, A., Bauer, J., Cutri, R. M., et al. 2014, ApJ, 792, 30

Masci, F. J., Laher, R. R., Rusholme, B., et al. 2019, PASP, 131, 018003

Megeath, S. T., \& Wilson, T. L. 1997, AJ, 114, 1106

Minniti, D., Lucas, P. W., Emerson, J. P., et al. 2010, NewA, 15, 433

Moore, A. M., \& Kasliwal, M. M. 2019, NatAs, 3, 109

Sato, M., Hirota, T., Honma, M., et al. 2008, PASJ, 60, 975

Shappee, B. J., Prieto, J. L., Grupe, D., et al. 2014, ApJ, 788, 48

Sharma, S., Pandey, A. K., Pandey, J. C., et al. 2012, PASJ, 64, 107

Skrutskie, M. F., Cutri, R. M., Stiening, R., et al. 2006, AJ, 131, 1163

Stecklum, B. 2020, ATel, 13834, 1
Szegedi-Elek, E., Ábrahám, P., Wyrzykowski, Ł., et al. 2020, ApJ, 899, 130 Tonry, J. L., Denneau, L., Heinze, A. N., et al. 2018, PASP, 130, 064505

Vacca, W. D., Cushing, M. C., \& Rayner, J. T. 2003, PASP, 115, 389

Werner, M. W., Roellig, T. L., Low, F. J., et al. 2004, ApJS, 154, 1

Wilson, J. C., Eikenberry, S. S., Henderson, C. P., et al. 2003, Proc. SPIE, 4841,451

Wilson, J. C., Henderson, C. P., Herter, T. L., et al. 2004, Proc. SPIE, 5492,1295

Wright, E. L., Eisenhardt, P. R. M., Mainzer, A. K., et al. 2010, AJ, 140, 1868

Zackay, B., Ofek, E. O., \& Gal-Yam, A. 2016, ApJ, 830, 27 